\title{
Some nonmasking auditory postsignal effects
}

\author{
RICHARD E. PASTORE and CHARLOTTE S. MacLATCHY \\ State University of New York at Binghamton, Binghamton, New York 18901
}

\begin{abstract}
Observers were asked to detect a 20 -msec segment of a sinusoidal signal masked by a band-limited white noise. A postsignal decrease in the spectrum level of the noise within the critical band of the signal enhanced the detectability of that signal if the decrease occurred within approximately $25 \mathrm{msec}$ following signal termination. Postsignal decreases outside the critical band of the signal, and decreases within the critical band delayed longer than approximately $40 \mathrm{msec}$, reduced the detectability of the signal for decrease delays up to between approximately 150 and $400 \mathrm{msec}$, depending on the spectral characteristics of the decrease. Comparisons with typical backward masking results indicate probable common factors of short-term temporal summation and longer term attention.
\end{abstract}

Sperling (1960. 1963) demonstrated the persistence and processing of visually presented information for a period of several hundred milliseconds following the termination of the brief physical signal. The integrity of the stored information was found to be susceptible to disruption by a postsignal masking (Baxt) stimulus. It is quite probable that there is an analogous short-term storage of information in the auditory system. ${ }^{1}$ The notion of the persistence and/or temporal smearing of auditory stimuli is supported by a number of tindings: the 10- to $15-\mathrm{msec}$ threshold for temporal gap detection (Abel, 1972); the 15- to 20 -msec interstimulus interval threshold for temporal order discrimination (Hirsh, 1959); the "universal" 20-msec boundary for the "categorical" perception of speech (Abramson \& Lisker, 1970; Mattingly, 1972) and complex nonspeech signals (Miller, Pastore, Wier, Kelly, \& Dooling, 1974); the \pm 100 -msec effective period for backward and forward masking stimuli (Elliott. 1971); and the $200-300$ msec required for an auditory sensation to reach threshold (Plomp, 1964). Wright (1964) hypothesized three stages to backward masking. These three stages are consistent with the various effects described above and include (1) an initial short phase of approximately $25 \mathrm{msec}$ in which the perceptibility of the first signal is a function of the parameters of the second signal. (2) a long phase at delays between approximately 50 and $300 \mathrm{msec}$ in which only the presence or absence of the second stimulus intluences the perception of the first stimulus, and (3) a transition phase between the short and long phases. The short phase is probably due to some form of energy summation. ${ }^{2}$ The factors underlying the longer phase are more obscure.

The studies cited above indicate that the addition of a second signal during the interval following a given

This research was supported by Research Grant MH 24508 from the National Institute of Mental Health. Requests for reprints should be sent to the first author in care of the Department of Psychology, SUNY-Binghamton, Binghamton, New York 13901. signal alters the perception of that first signal and any "silent" interval following that signal. The major aspect of the second signal causing these effects could be (1) the additional energy supplied by the second signal, (2) the change (increase or decrease) in energy level caused by the addition of the second signal, or (3) both. Green and Sewall (1962) have found that human observers better detect noise bursts masked by continuous rather than gated masking noise in a two-alternative forced choice paradigm. [This superiority of gated maskers is not always found (i.e., Tucker, Williams, \& Jeffress, 1968; Campbell, 1969)]. Green (1969) also reported greater simultaneous masking of a sinusoidal signal by a sinusoidal masking stimulus when the masker was gated rather than continuous. The superiority of the gated sinusoidal masker was approximately $5 \mathrm{~dB}$ when the masker and signal were similar in frequency and was up to $40 \mathrm{~dB}$ when their frequencies differed. While Green favored an energy splatter explanation of his data, he ack nowledged the possible significance of the simple change in the stimulus situation. Stimulus changes are known to produce greater neural firing (Kiang, 1965) and greater psychophysical masking (Elliott. 1965; Leshowitz \& Cudahy, 1972; Zwicker, 1965) than stimuli which have been stationary for a short period of time. If a simple change in the stimulus configuration has a disrupting or masking effect on a signal in close temporal proximity, then a change in the form of the removal of energy from an otherwise continuous masking noise should increase rather than decrease the disruptive effects of the noise on an embedded signal.

Two series of signal detection experiments were conducted. All involved the detection of brief segments of a sinusoidal signal. The experiments investigated the effects of a reduction in the spectrum level of selected portions of an otherwise stationary masking noise. Both the frequency limits of the spectral decrease and the delay between the end of the 
observation interval and the onset of the spectral decrease were varied. It was hypothesized that if the short phase of backward masking involves some form of temporal summation. an immediate poststimulus reduction in the masking energy within the critical band of the signal should enhance the detectability of the signal relative to the control condition in which the noise is not changed. Furthermore, if the long phase of backward masking involves the effects of a simple change in the stimulus, the delayed reduction within the critical band of the signal and any reduction outside the critical band of the signal should have a disrupting effect on detection performance relative to the stationary control condition.

\section{METHOD}

Subjects

All three observers had clinically normal hearing and were paid for their participation in the study. The obseners were run either singly or in pairs. always with each observer in a separate sound-insulated chamber.

\section{Procedure}

The observers were asked to detect. in a standard yes-no paradigm. a monaural $20-\mathrm{msec}$ segment of a $1.000-\mathrm{Hz}$ sinusoid gated at positive waseform zero-crossings. The signal was generated by a Hewlett-Packard 204D oscillator, gated by an analog switch. attenuated. then presented to the observers through Grason-Stadler TDH $-39(300)$ earphones with a probability of 0.50 . The masking noise had two components which were generated by separate General Radio 1382 noise generators. The variable noise component (see Figure 1) simultaneously was ted directly to one electronic switch $\left(N_{V}\right)$ and through a Krohn-Hite 3750 filter to a second switch $\left(N_{F}\right)$. The outputs of these two switches were mixed with the attenuated $\left(-15 \mathrm{~dB}\right.$ re: $\mathrm{N}_{V}$ component) output of the other noise generator ( $\mathrm{NC}$ ), fed through an Allison 2BR filter (360) to $3.300 \mathrm{~Hz}$ ). and mixed with the output of the signal switch. One control and two experimental conditions were run. In the control condition, the masking noise was continuous and consisted of the batnd-limited mixture of $N_{C}$ and $N_{V}$. The experimental conditions differed from the control condition only by a change from $N_{V}$ to $N_{F}$ initiated $\Delta t$ msec after the end of the observation interval and lasting $1.000 \mathrm{msec}$. Thus, the spectrum level of the masking noise was decreased by $15 \mathrm{~dB}$ in a limited frequency region for $1.00 \mathrm{sec}$ of each 3.00-sec trial. beginning $\Delta t$ msec after the end of the observation interval. The spectrum level of the masking noise remained unchanged in all other frequency regions. In the band-pass condition. the spectrum level of the noise was decreased at all frequencies texept between $7 \overline{0})$ and $1.400 \mathrm{~Hz}(-3 \mathrm{~dB}$ points measured through whole audio circuit). In the band-reject condition. the spectrum level was decreased only between 700 and 1. $f(x) \mathrm{Hz}$. During the observation interval, the masking noise had an overall level of $68 \mathrm{~dB}$ re: 0.0002 microbar at the output of the earphone under all conditions. In summary, the noise was identical on both experimental and control trials, except for a 1 -sec period initiated $\Delta t$ msec following the end of the observation interval. During this 1 -sec period, the noise (1) remained unchanged (control condition). (2) was reduced onls in the frequency region of the signal (band reject). or (3) was reduced everywhere except in the frequency region of the signal (band pass).

The observers ran in blocks of 100 continuous trials. During each block of trials. onls one condition with a single delas. $\Delta t$. was in effect. Blocks of control condition trials were mixed with blocks of a single experimental condition with a tixed $\Delta t$ during any one session. ${ }^{3}$ When beginning the new experimental condition (BR. BP). the tirst several hours were treated as practice. The tirst hour at each delay. $\Delta t$. also was treated as practice because of an a priori concern that these data might be contaminated by learning effects. A total of 1.500 experimental trials of data per observer were collected at each of two signal levels at each delay under a given experimental condition betore the next, longer delay was imposed. Generally: all data under a given experimental condition were collected for a given observer betore the observer changed to another experimental condition.

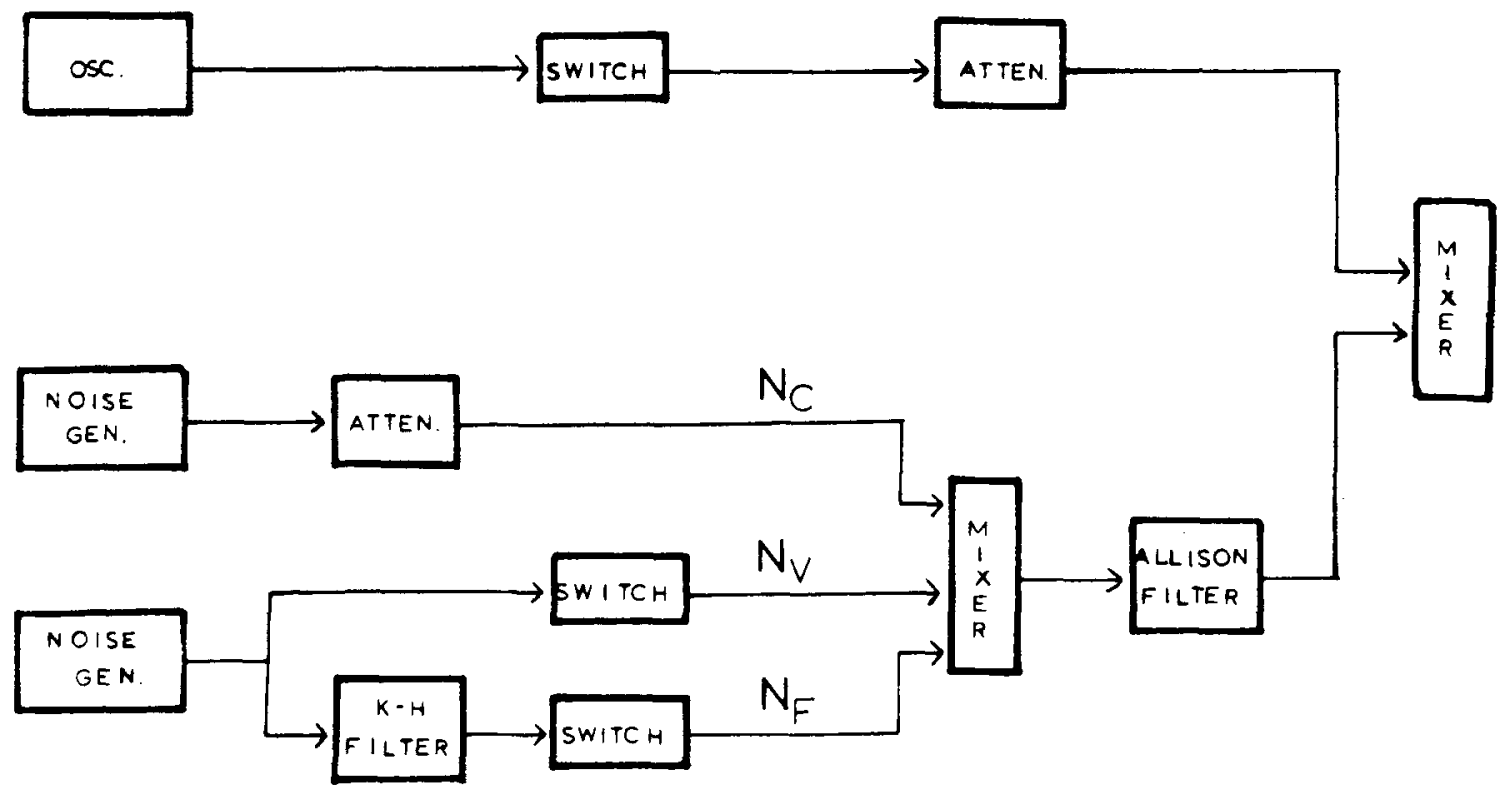

Figure 1. Block diagram of the equipment used to generate the signals (see text for description). 


\section{RESULTS}

A value of d' was computed for each block of 100 trials. For each observer, the mean value of the $15 \mathrm{~d}^{\prime}$ measures for each delay/condition was computed. The difference, $\Delta d^{\prime}$, between each mean value of $d^{\prime}$ and the mean value of $d^{\prime}$ for the control condition run in the same block of trials is plotted in Figures $2 a$ and $2 b$ as a function of $\Delta t$ for the three observers under the band-reject conditions at each of the two signal-to-noise ratios. ${ }^{4}$ The values of $10 \log \mathrm{E} / \mathrm{N}_{\mathrm{O}}$ are shown on the figures. The average value of $d^{\prime}$ for the control condition ranged from 1.4 (Obs. 4) to 1.9 (Obs. 3), and from 2.3 (Obs. 4) to 2.8 (Obs. 3) for the two signal-to-noise ratios. Under the various experimental conditions, the individual values of $d^{\prime}$ across delays and observers ranged from 0.5 to 2.1 and from 1.1 to 2.9 for the two signal-to-noise ratios. ${ }^{5}$

The trends in the data for the three observers are highly similar. These data all indicate that a poststimulus decrease in the spectrum level of the noise in the frequency region of the signal improves the detectability of that signal if the decrease cccurs within 10 to $40 \mathrm{msec}$ following the end of the observation interval. The duration of this short poststimulus phase may be related (inversely) to the detectability of the signal. Following this short period, there is a longer period, lasting several hundred milliseconds, during which the same decrease in the spectrum level of the noise interferes with the detectability of the signal.

Figure 3 shows the effects of a poststimulus decrease in the spectrum level of the masking noise confined to the frequency regions outside the critical band for the signal. The data for the two signal-to-noise ratios indicate that this poststimulus change reduces the detectability of the signal; these effects appear to follow a general, skewed. U-shaped trend as a function of the delay, $\Delta t$. The effects are minimal at extremely short delays $(1 \mathrm{msec})$ and at relatively long delays $(\Delta t>150 \mathrm{msec})$. A comparison of the data for the two signal levels (Figures $3 a$ and $3 b$ ) would seem to indicate that the effects of the poststimulus change are greater for the more detectable signals, although this simply may reflect a greater range for potential change at the higher level of performance.

The similarities in the various functions across observers are clear. The typical standard deviations of the $d^{\prime}$ values for each observer/delay/condition was approximately 0.3 for both experimental and control data. The 2-dB change in the signal-to-noise ratio for the control condition caused a change in $d^{\prime}$ of approximately 0.70 . Therefore, the maximum $\Delta \mathrm{d}^{\prime}$ values represent an equivalent 2 - to $4-\mathrm{dB}$ change in signal level, with a 4 - to $7-\mathrm{dB}$ range between the positive and negative peaks of the function for the band-reject condition.
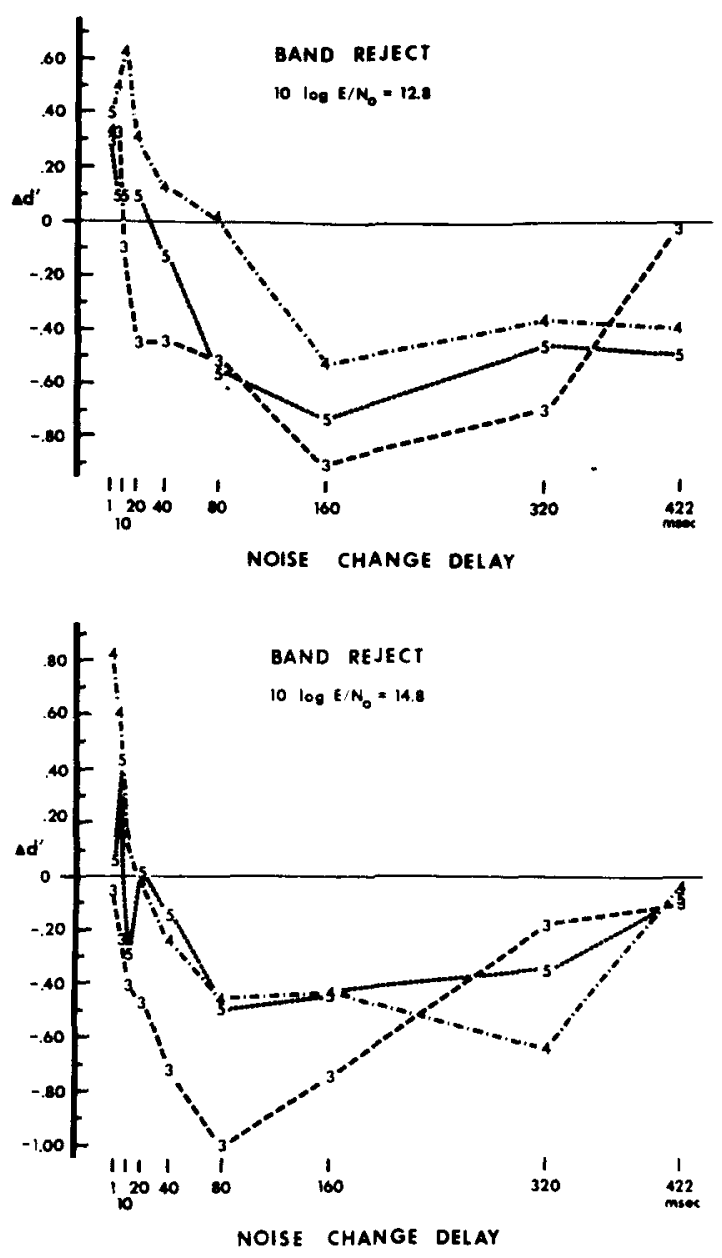

Figure 2. $\Delta d^{\prime}$ (differences in d' between experimental and control conditions in a given set of sessions) as a function of change delay for spectral decreases in the frequency region of the signal. The data in Figure $2 a$ are based on mean values of $d^{\prime}$ under the control condition of 1.9, 1.4, and 1.7 for Observers 3, 4, and 5, respectively. The values of the control condition $d^{\prime}$ were $2.8,2.3$, and 2.6 , respectively, for the data plotted in Figure $\mathbf{2 b}$.

\section{DISCUSSION}

The data all indicate that a poststimulus change (decrease in spectrum level) of an otherwise continuous masking noise affects the detectability of the signal. If the poststimulus decrease is confined to the frequency region of the signal, a decrease within the first 20 to $40 \mathrm{msec}$ following the end of the observation interval will enhance the detectability of the signal, while a decrease initiated after approximately 30 to $400 \mathrm{msec}$ will reduce the detectability of the signal. These results are theoretically consistent with the three stages of backward masking described by Wright (1964). A decrease contined to the frequency region outside the critical band of the signal will reduce the detectability 


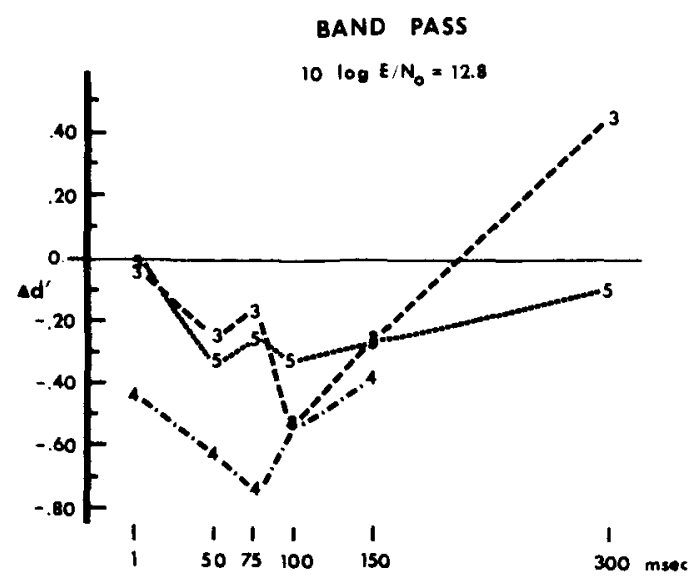

NOISE CHANGE DELAY

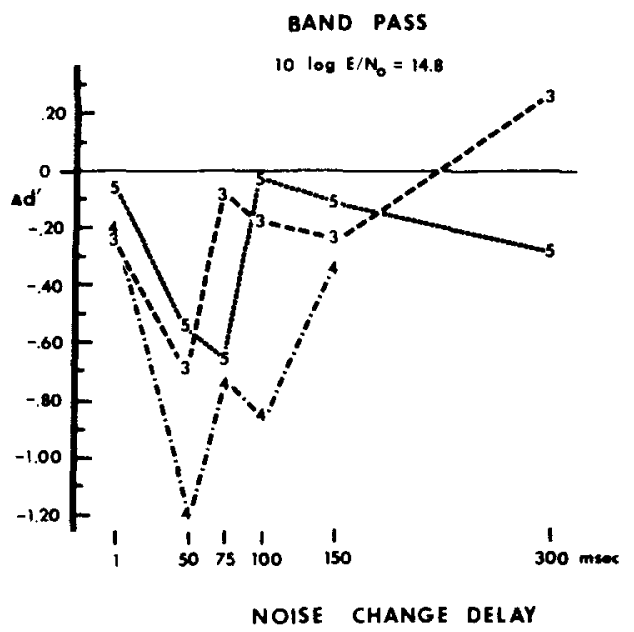

Figure 3. $\Delta d^{\prime}$ as a function of change delay for changes not involving the signal frequency region. Figures $3 a$ and $3 b$ show the data for $10 \log E / N_{0}$ equal to 12.8 and 14.8 , respectively. The mean values of $d$ for the control conditions are specified under Figure 2.

of the signal, with the maximum reduction being reached at approximately $80 \mathrm{msec}$ and with little effect after approximately 100 to $150 \mathrm{msec}$.

The difference between a within-critical-band and an extra-critical-band short-delay decrease in the masking noise is consistent with the temporal summation explanation of backward masking proposed by Wright (1964), Zwislocki (1960), and others. If the observer integrates the stimulus waveform over a temporal interval which is longer than the observation interval, the within-critical-band poststimulus decrease in the masking noise would reduce the total effective level of the masking noise and thus increase the effective signal-to-noise ratio relative to the control condition. Since only energy within the critical band of a signal is effective in masking that signal (e.g., Fletcher, 1940), an extra-critical-band poststimulus decrease should not alter the effective signal-to-noise ratio.
The long-term interference effects noted for any type of change in the poststimulus masking noise probably are due to causes not related to a simple summation of energy. The U-shaped functions for the band-pass condition might indicate that there are initially two, possibly opposing. processes involved: a short-term enhancement and a long-term interference. The use of a temporally tixed poststimulus change could have provided the observer with precise temporal information not available with the visual cue defining the observation interval. Such information could be utilized to terminate more precisely an integration process, or to define more precisely the temporal segment of the continuous waveform which could contain the signal. Either mechanism would effectively reduce the total masking noise entering the decision process (e.g., Green \& Sewall, 1962), and would be useful only if the change occurred within a short time following the observation interval.

There are a number of possible explanations for the long-term interference effects. If one assumes (1) that the system is processing a stored representation of a temporal segment of the waveform, and (2) that an abrupt, detectable stimulus change is processed with a higher probability than a change which is "near threshold," the poststimulus change would have caused the system to process the wrong information. Temporal and spatial changes (or borders) are known to be significant factors in visual perception (Gregory, 1973; Ratliff, 1965) and, when viewed in the context of adaptation, in most senses (Geldard, 1972) and in attention (Mostofsky, 1970). There also is evidence, though very limited, for an auditory information storage similar to that proposed by Sperling for vision (e.g., Treisman \& Rostron, 1972). A more parsimonious explanation. which encompasses the above, is to assume that the postsignal change is a distractor, diverting "attention" from the appropriate "decision process." A third hypothesis is based on the assumption that the decision process involves a comparison of the parameters of the waveform temporally defined by the visual cue with some estimate of the average parameters of the noise based on sampling the temporal period following the observation interval. Currently, there are insufficient data to evaluate the various possible hypotheses and conjectures.

\section{REFERENCES}

ABEL, S. M. Discrimination of temporal gaps. Journal of the Acoustical Society of America, 1972, 52, 519-524.

Abramson, A. S., \& Lisker, L. Discriminability along the voicing continuum: Cross-language tests. In Proceedings of the Sixth Intemational Congress of Phonetic Sciences. Prague: Academia, 1970.

Campbell, R. A. Thresholds re duration and levels of a continuous or gated masker. Journal of the Acoustical Society of America, 1969, 46, 895-897. 
Darwin, C. J., Turvey, M. T., \& Crowder, R. G. An auditory analogue of the Sperling partial report procedure: Evidence for brief auditory storage. Cognitive Psychology, 1972, 3. $255-267$.

ElLIOTT. L. L. Changes in the simultaneous masked threshold of briet tones. Journal of the Acoustical Society of America, 1965. 38. 738-746.

Elf.iott, L. L. Backward and forward masking. Audiology, $1971,10,65-76$.

Fletcher, H. Auditory patterns. Reviews of Modern Physics, $1940,12,47-65$.

Geldard, F. A. The human senses. New York: Wiley, 1972.

GrEEN, D. M. Masking with continuous and pulsed sinusoids. Journal of the Acoustical Society of A merica, 1969, 46, 939-946.

GreEN, D. M., \& SEwall, S. T. Effects of background noise on auditory detection of noise bursts. Journal of the Acoustical Society of America, 1962. 34. 1207-1216.

GREGORY, R. L. Eye and brain: The psychology of seeing. New York: McGraw-Hill, 1973.

HIRSH, I. J. Auditory perception of temporal order. Journal of the Acoustical Society of America, 1959, 31, 759-767.

KIANG. N. Y.-S. Discharge patterns of single fibers in the cat's uuditony nen'e. Cambridge, Mass: M.I.T. Press, 1965.

Leshowitz. B., \& Cudahy, E. Masking with continuous and gated sinusoids. Journal of the Acoustical Society of America, 1972, 51, 1921-1928.

Mattingly, I. G. Speech cues and sign stimuli. American Scientist, 1972.60. 327-337.

Miller. J. D.. Pastore, R. E., Wier, C. C., Kelly, W. J., \& Dooling. R. J. Discrimination and labeling of noise-buzz sequences with varying noise lead times. Journal of the Acoustical Society of A merica, 1974, 55, 390(A).

Mostofsky, D. I. (Ed.) Attention: Contemporary theory and analysis. New York: Appleton-Century-Crofts, 1971.

PASTORE, R. E. Some possible attentional aspects of post-stimulus signal processing. Joumal of the Acoustical Society of America, 1973, 53, 376(A).

Plomp, R. Rate of decay of auditory sensation. Journal of the Acoustical Society of America, 1964, 36, 277-282.

RATLIFF, F. Mach bands: Quantitative studies on neural networks in the retina. San Francisco: Holden-Day, 1965.

SPERLING, G. The information available in brief visual presentations. Psychological Monographs, 1960, 74(11, Whole No. 498).

SPERLING, G. A model for visual memory tasks. Human Factors, $1963,5,19-31$.
Treisman, M., \& Rostron, A. B. Brief auditory storage: A modification of Sperling's paradigm applied to audition. Acta Psychologica, 1972, 36, 161-170.

Tucker, A., Williams, P. J., \& Jeffress, L. A. Effect of signal duration on detection for gated and for continuous noise. Joumal of the Acoustical Society of America, $1968,44,813-816$.

WRIGHT, H. N. Temporal summation and backward masking. Journal of the Acoustical Society of A merica, 1964, 36, 927-932.

ZwICKER, E. Temporal effects in simultaneous masking by whitenoise bursts. Journal of the Acoustical Society of America, $1965,37,653-663$.

ZWISLOCKI, J. Theory of temporal auditory summation. Journal of the Acoustical Society of America, 1960, 32, 1046-1060.

\section{NOTES}

1. Two recent studies (Darwin, Turvey, \& Crowder, 1972; Treisman \& Rostron, 1972) have attempted to apply Sperling's cueing procedure to investigate short-term auditory information storage. These studies investigated much longer temporal effects $(400-4,000 \mathrm{msec})$ than the present study.

2. An explanation based on the differential transmission of stinuli of differing intensity seems inadequate to explain all of the cited short-term $(\Delta t<25$ msec) phenomena.

3. Blocks of 150 trials also were run in which there was an equal probability of the control condition and a given experimental condition on every trial. While the data for the two types exhibited the same trends noted for the separate blocks of trials, the additional uncertainty in the trial structure reduced the level of performance of the observers. This procedure therefore was abandoned in tavor of running separate blocks of trials.

4. The data for two other observers (Obs. 1 and 2) under the band-reject and band-pass conditions were presented to the 86 th meeting of the Acoustical Society of America (Pastore, 1973), and are similar to these data.

5. A listing of the mean and standard deviation for each condition/delay/observer may be obtained by writing the authors.

(Received for publication August 19, 1974; revision received January 10,1975 .) 